\title{
LA ENSEÑANZA DE LAS CIENCIAS SOCIALES EN LA ESCUELA PRIMARIA ${ }^{1}$
}

\author{
THE TEACHING OF SOCIAL SCIENCES IN THE ELEMENTARY SCHOOL
}

\author{
María Eugenia Pérez-Guzmán²*. \\ 2. Universidad de Antioquia, Medellín, Colombia. maria16071@hotmail.com \\ *Autor de correspondencia: María Eugenia Pérez Guzmán, correo electrónico: maria16071@hotmail.com
}

\section{RESUMEN}

El presente artículo de revisión, tiene como objetivo identificar qué se ha venido investigando sobre la enseñanza de las Ciencias Sociales en la básica primaria, a partir del rastreo de artículos de revistas derivados de investigaciones, estudios de maestría y doctorado, en países como España, México, Chile, Argentina y Colombia en los últimos cincos años. Con dicha revisión se logró concluir que los estudios sobre la enseñanza de las Ciencias Sociales están enfocados en aspectos didácticos e históricos. De ahí que es importante propender por investigaciones que busquen indagar cómo se concreta la enseñanza de las Ciencias Sociales en la escuela primaria, para tener una mirada global del objeto de estudio.

Palabras clave: Enseñanza de las Ciencias Sociales; didáctica de las Ciencias Sociales; configuración de la enseñanza; escuela primaria; concepciones; guía de aprendizaje.

\section{Cómo citar:}

Pérez-Guzmán, María Eugenia. (2021). LA ENSEÑANZA DE LAS CIENCIAS SOCIALES EN LA ESCUELA PRIMARIA. Revista de Investigaciones Universidad del Quindio, 33(2), 20-31. https://doi.org/10.33975/riuq.vol33n2.546

Información del artículo: Recibido: 7 agosto 2021; Aceptado: 19 agosto 2021

\footnotetext{
1 El artículo se deriva del proyecto de investigación titulado: Configuración de la enseñanza de las Ciencias Sociales en tres instituciones del municipio de Medellín.
} 


\begin{abstract}
The objective of this review article is to identify what has been researching about the teaching of Social Sciences in elementary school, based on the tracking of articles from journals derived from research, master's and doctoral studies, in countries such as Spain, Mexico, Chile, Argentina and Colombia in the last five years. With this review, it was concluded that, the studies on the teaching of the Social Sciences are focused on didactic and historical aspects. Therefore, it is important to promote research that seek to investigate how the teaching of the Social Sciences in primary school is realized to have a global view of the object of study.
\end{abstract}

Keywords: Social Sciences teaching; Social Sciences didactics; teaching configuration, primary school, conceptions; learning guides.

\section{TENSIONES EN LA ENSEÑANZA DE LAS CIENCIAS SOCIALES EN LA BÁSICA PRIMARIA.}

El artículo al tener como objetivo identificar en los últimos cinco años que se ha investigado sobre la enseñanza de las Ciencias Sociales en la básica primaria, debe retomar las prácticas de enseñanza, es decir, si en la primaria sobresalen las prácticas de enseñanza atravesadas por las clases magistrales, actividades propuestas en libros de textos, el asignaturismo y docentes con poca formación en el área, o si, por el contrario, se plantean procesos dialógicos y reflexivos con el fin de formar sujetos críticos.

De igual modo, entender de qué modo la enseñanza de las Ciencias Sociales está marcada por reformas curriculares y estas como inciden en las prácticas de enseñanza de los maestros de básica primaria, puesto que no son disposiciones neutras, lo que tensiona la autonomía, libertad de cátedra y enseñanza contextualizada. También, es importante tener presente la existencia de noción de Ciencias Sociales integradas y como esto ha repercutido en las prácticas cotidianas y finalmente reconocer como la enseñanza de las Ciencias Sociales se pueden concretizar mediante dispositivos como las guías de aprendizaje.

\section{CRITERIOS DE BÚSQUEDA.}

Para abarcar el objeto de estudio se realizó un rastreo de los últimos cincos años en: artículos de revistas derivados de investigaciones, estudios de maestría y doctorado, países como España, México, Chile, Argentina y Colombia, bases de datos como DOAJ, REDALYC, DIALNET, SCIELO, Revista Colombiana de Educación, Revista Pedagogía y Saberes, Revista MAGIS, Revista Unipluriversidad y Revista Enseñanza de las Ciencias Sociales. Dicha investigación tuvo como propósito conocer: la enseñanza de las Ciencias Sociales en primaria, historia de la enseñanza de las Ciencias Sociales en primaria, los maestros de Ciencias Sociales en primaria, guía de aprendizaje en la enseñanza y aprendizaje, historia del currículo de las Ciencias Sociales en primaria y currículo de la enseñanza de las Ciencias Sociales.

Con la información seleccionada se logró establecer categorías como: maestros de Ciencias Sociales y sus prácticas de enseñanza, prácticas de enseñanza en las distintas disciplinas sociales, reformas curriculares que permean la enseñanza de las Ciencias Sociales en Colombia desde un enfoque histórico, concepciones de la enseñanza de las ciencias sociales en educación primaria en Colombia desde los referentes de calidad y la guía de aprendizaje como dispositivo donde se materializa la enseñanza de las Ciencias Sociales, las cuales se desarrollan a continuación:

Maestros de Ciencias Sociales y sus prácticas de enseñanza.

En esta categoría se encuentran los trabajos 
sobre las prácticas de enseñanza de los maestros de Ciencias Sociales en la básica primaria, con el objetivo de conocer qué posturas pedagógicas y qué estrategias didácticas predominan en su praxis.

En este orden de ideas, Tosar (2018), durante una investigación que realizó en España, pretendió interpretar diferentes tipos de textos relacionados con las Ciencias Sociales. Sus hallazgos fueron que la enseñanza en la educación primaria debe promover los contenidos a través de la lectura de todo tipo de textos, imágenes y discursos, para así formar personas reflexivas capaces de interpretar la realidad que habita, razón por la cual, a los maestros en clase les corresponde abordar fenómenos con múltiples perspectivas.

Por su parte, Moreno (2018), realiza un estudio para analizar la forma en la que el profesorado de educación primaria de España concibe las Ciencias Sociales escolares y su enseñanzaaprendizaje a partir de recuerdos, reflexiones y expectativas. En los resultados que obtiene, el autor muestra que predomina la metodología tradicional fundamentada en clases magistrales, actividades basadas en libros de texto, contenidos históricos netamente teóricos $\mathrm{y}$ contenidos geográficos que ofrecen herramientas procedimentales. Evidenciándose así que la reflexión crítica que se debe fomentar en los primeros años de escuela no se lleva a cabo, dado que los maestros en ejercicio se quedan en la anécdota, pues su formación no les permite analizar de forma crítica la realidad social, económica, política y curricular que atraviesa su práctica de enseñanza.

Asimismo, un estudio realizado por Díaz (2017), en España, tuvo como finalidad analizar las estrategias de enseñanza y aprendizaje que se emplean en el contexto del aula, donde encontró que enseñar los contenidos sociales a partir de problemáticas cotidianas posibilita la adquisición de competencias necesarias para vivir en sociedad, lo que hace indispensable que en primaria se aborden los problemas sociales relevantes con la intención de analizarlos y descubrir posibles soluciones que conduzcan al cambio social. Por eso, es de vital importancia que los docentes motiven a los estudiantes e incentiven el pensamiento reflexivo, social y crítico por medio de estrategias didácticas.

En cambio, Ortega (2015), se enfocó en analizar las relaciones inherentes entre la enseñanza de la Historia de Iberoamérica y la construcción de identidades culturales por medio del estudio normativo comparado en España, México y Chile de los libros de texto, como uno de los materiales de concreción curricular en el ámbito educativo. En este se concluyó que los libros de textos utilizados en las clases de Ciencias Sociales en Iberoamérica, utilizan como eje central la Historia para reproducir las concepciones de los estados-naciones y el modelo de ciudadano a formar. Se resalta también que los libros de texto son usados principalmente en las escuelas primarias.

Otro antecedente es la investigación realizada por Amézola y D'Archary (2016), quienes indagaron por la dictadura militar y cómo esta se instaló en las escuelas públicas primarias del partido de Malvinas Argentinas en la Provincia de Buenos Aires, a través de las conmemoraciones y las actividades. Dichas autoras visualizaron que en las clases se utilizaban estrategias didácticas como: investigación de la realidad, relatos y entrevistas a familiares, análisis de literatura recomendada y censurada en ese momento histórico, canciones, análisis audiovisuales, debates, lectura de documentos históricos, lectura de cuentos, observación de imágenes, mapas y fotos acordes al momento histórico, búsqueda en internet, estudio de libros otorgados por el Ministerio de Educación de la Nación Argentina. No obstante, se señala que cuando las estrategias están muy enfocadas en los alumnos es porque el docente tiene poca preparación o dominio conceptual de estas temáticas, lo que causa que se apoye en el alumno para que sea este quien construya un hecho y sus consecuencias. 
Finalmente, Gutiérrez y Arana (2014), se proponen identificar, caracterizar e interpretar las habilidades de pensamiento social a partir del estudio de ocho experiencias de enseñanza y aprendizaje de las Ciencias Sociales con estudiantes de educación básica primaria de la ciudad de Pereira- Colombia. En este estudio se visualizó que las Ciencias Sociales en primaria no contribuyen a la formación de este tipo de pensamiento, por lo cual es urgente que las prácticas educativas sean reflexivas, basadas en el diálogo y la interacción social. Dicho de otro modo, los maestros no solo deben ser quienes dicen qué y cómo abordar el conocimiento social, sino que deben fomentar la interacción social para potencializar habilidades como la atención, la imaginación, la inferencia, etc., las cuales ayudan a la construcción de significados y su utilización en el medio social, que es la finalidad de la enseñanza de las Ciencias Sociales en primaria.

\section{Prácticas de enseñanza en las distintas disciplinas sociales.}

En este apartado aparecen los trabajos que retoman la figura de los maestros de básica primaria desde diferentes disciplinas de las Ciencias Sociales, con el objetivo de visibilizar cómo llevan a cabo sus prácticas de enseñanza.

En esta línea de sentido, Sáez-Rosenkranz(2017), quiso identificar y analizar los aprendizajes presentes en las actividades de los libros de texto de educación básica en Chile bajo el currículum vigente, hallando que algunos textos mantienen miradas tradicionales en la enseñanza de historia. Por eso se considera que los estudiantes deben aprender hechos significativos del devenir histórico, y a su vez tener herramientas para enfrentar de forma crítica la realidad mediante la formulación de preguntas acerca del pasado y del presente que promuevan la apropiación de conceptos como tiempo, cambio, continuidad, causalidad-consecuencia y la relación con el presente. Lo que incidiría en que los estudiantes tuvieran la capacidad de enfrentarse a fenómenos sociales desde una mirada analítica, para comprender y valorar su propio contexto histórico.

A su vez Binimelis y Ordinas (2016), tuvieron como propósito indagar por los conocimientos mínimos de geografía exigibles a los futuros docentes a través de una prueba de conocimientos basada en el currículo de Ciencias Sociales de educación primaria de la Comunidad Autónoma de las Islas Baleares- España. Esta indagación permitió descubrir que las reformas curriculares españolas han sido constantes, lo que pone en tela de juicio la solidez y rigurosidad de dicha área, de igual modo, se visualiza que se ha dejado por fuera la enseñanza de la geografía, la cual aporta conceptos y procedimientos importantes para la educación primaria y el entendimiento del mundo. A lo anterior se le suma que en la formación de docentes de primaria no se alcanza a interiorizar los contenidos propios de la disciplina en la que se forma, en este caso la geografía.

Por otro lado, Pinto y Molina (2015), estudiaron el caso de la educación primaria en España y la educación elemental en Portugal, con el fin de analizar cuál es el concepto de patrimonio propuesto en las nuevas regulaciones, qué ámbitos del patrimonio están siendo priorizadas y qué objetivos son perseguidos por la educación patrimonial en estos nuevos textos legales. En dicha investigación se encuentra que, en el caso español, a través de la propuesta LOMCE (La Ley Orgánica 8/2013 del 9 de diciembre para la mejora de la calidad educativa), desaparece el área de Conocimiento del Medio Natural, Social y Cultural, en la que se impartían contenidos propios de Ciencias Sociales y de Ciencias Naturales. (Miralles, 2009, citado en Pinto y Molina 2015), y se crean dos áreas diferenciadas: la de Ciencias Naturales y la de Ciencias Sociales, esta última encargada de impartir conocimientos geográficos, sociales, económicos, políticos e históricos. En el caso de Portugal a través del Decreto-Lei n. ${ }^{\circ}$ 91/2013 del 10 de julio y el área curricular de Estudo do 
Meio de 1998, se señala que dicha área maneja conceptos y métodos propios de la Historia, la Geografía, las Ciencias Naturales y la Etnografía, para establecer relaciones entre la naturaleza y la sociedad. De la misma manera, se distingue que el patrimonio ofrece un gran potencial didáctico para la enseñanza de las Ciencias Sociales, no obstante, su presencia en las prácticas de enseñanza aún es incipiente, parcial y limitada.

Igualmente, Gómez, et al (2015), buscaron demostrar el potencial didáctico de los nombres de lugar para explicar la diversidad del territorio y hacer emerger lugares vulnerables o sometidos a riesgos; lugares que deben ser objeto de aprendizaje en nuestro campo de conocimiento y recibir atención en los procesos de enseñanzaaprendizaje de las Ciencias Sociales en educación primaria. Este estudio encontró que se debe aprovechar el potencial que tiene el concepto de topónimos para el análisis territorial, puesto que es un asunto de interés social que contempla aspectos como la fragilidad, los riesgos, la ocupación y la explotación a la que se enfrentan los territorios, lo que se puede convertirse en una manera de promover la curiosidad del alumnado. Hay que mencionar además que Hernández (2014), analizó los elementos que permitieran relacionar: a) la enseñanza de las Ciencias Sociales, b) la enseñanza del tiempo histórico y c) la socialización. Lo que permitió concluir que aunque las teorías piagetianas consideran que los niños no tienen capacidad de hacer abstracciones relacionadas con el tiempo y el espacio, investigaciones sobre la enseñanza de la Historia en los primeros años de escuela han demostrado que los niños comprenden diferentes conceptos relacionados con el pensamiento histórico; de ahí surge la importancia de potencializar estos pensamientos a partir de actividades que involucren la creatividad, la imaginación y el interés. Lo anterior indica que la enseñanza de la Historia en la primaria tiene la finalidad de formar el sentido del tiempo y el pensamiento histórico, razón por la cual los profesores no deben enseñar los acontecimientos del pasado sino generar reflexiones y análisis sobre ellos.
Por su parte, en el contexto chileno, Pinochet y Pagés (2016), interpretaron los propósitos presentes en las programaciones ministeriales de las asignaturas de Historia, Geografía y Ciencias Sociales, así como en los discursos de los estudiantes con respecto al protagonismo de niños y jóvenes en la historia, donde encontraron que aunque las políticas chilenas habían dado un lugar a los niños, esto no se veía reflejado en la escuela, pues en clases de Historia se limitaba a hablar sobre los grandes personajes políticos por medio de los libros de texto, lo que convertía al estudiante en un sujeto pasivo; en otras palabras, la historia escolar se encuentra totalmente desvinculada de la realidad en la que se desenvuelven los estudiantes cotidianamente. Por eso se concluye que el currículo de Historia, Geografía y Ciencias Sociales de Chile, es un currículo en el cual coexisten dos miradas: 1) una mirada innovadora del por qué y para qué se debe estudiar Historia, 2) una mirada tradicional que promueve la enseñanza de la Historia de los de siempre. Sin embargo, y a pesar de existir dos opciones, el docente privilegia la enseñanza de la historia tradicional, donde el niño queda sumido en un rol pasivo.

Por último, en esta categoría, se encuentra la investigación de Duque (2017), quien se trazó el propósito de comprender el conflicto social en Colombia con base en 33 libros de textos escolares de Ciencias Sociales de básica primaria, publicados en el período 2003-2013. En esta investigación fue posible identificar que los libros de texto son un medio para estructurar el currículo, dado que muestra información sobre lo académico, cultural, educativo y político, con el fin de crear una percepción en quien lo estudia. En el caso de las Ciencias Sociales, los libros de textos de primaria reproducen el conflicto desde una concepción violenta y con figuras antagónicas, de modo que es necesario que estos libros reconozcan las narrativas de la realidad de los maestros y estudiantes, para que la enseñanza se oriente bajo problemas sociales relevantes.

En concordancia con lo anterior se puede 
identificar que las prácticas de enseñanza de las disciplinas sociales se encuentran centradas en la historia y la geografía, asimismo que las reformas curriculares que se han realizado en los distintos países, aunque propenden por una enseñanza crítica, aún no han trascendido de lo tradicional, lo cual incide en el poco aprovechamiento didáctico de los conceptos propios de las disciplinas abarcadas.

Reformas curriculares que permean la enseñanza de las Ciencias Sociales en Colombia desde un enfoque histórico.

En este punto se recogen las investigaciones que tienen por objeto de estudio las reformas curriculares para la enseñanza de las Ciencias Sociales en Colombia, esto con el fin de comprender desde qué enfoque se han hecho $\mathrm{y}$ en qué aspectos es necesaria una mayor profundización.

En su investigación Silva (2018), buscaba determinar el proceso de configuración de la enseñanza de las Ciencias Sociales como campo de saber -poder en Colombia y las condiciones y confluencias de luchas que permitieron la consolidación de dicho dominio en el periodo comprendido entre 1976 y 1994. El estudio plantea que el proceso de configuración de las Ciencias Sociales se da en cuatro periodos: el primero de 1936-1948, cuando las Normales Superiores comienzan a entregar el título de licenciados de la educación con especificidad en Ciencias Sociales, lo cual quiere decir que son maestros para la secundaria, asimismo, este periodo se ve atravesado por una ruptura entre la educación civilizatoria y la desarrollista; el segundo toma los años 1948-1976, en estos momentos el área se nombra Estudios Sociales y las reformas políticas y educativas incluyen las ciencias modernas y un enfoque científico; el tercero va desde 1974 hasta 1994, en el cual las Ciencias Sociales toman como eje una concepción internacionalista; y finalmente el periodo de 1994-2014, que está regulado por las dinámicas del mercado, la estandarización y la evaluación por competencias, lo que toma forma en los Lineamientos de Competencias de Ciencias Sociales (MEN, 2002) y Estándares Básicos de Competencias de Ciencias Sociales (MEN, 2004).

Acorde con ello, Velasco (2017), se preguntó ¿de qué manera las reformas al currículo oficial incidieron en el proceso de configuración de las Ciencias Sociales escolares en Colombia desde 1939 hasta 1974? Y encuentra que los planes y programas de estudio tienen un enfoque prescriptivo para formalizar el currículo de las áreas y asignaturas a través de contenidos de enseñanza, es decir, que son una expresión esquemática y lineal del proyecto de formación que ha construido como válido el sistema de educación nacional, además, es una manera de reafirmar la autoridad del Estado a través de la definición de los contenidos, y por tanto, se convierte en una forma de ejercer control.

Por otra parte, Aguilera (2016), revisó críticamente algunos lugares y supuestos que se dan por ciertos en el campo de la enseñanza de la Historia y las Ciencias Sociales en Colombia, con el fin de buscar alternativas en la enseñanza de estas disciplinas. Allí encontró que la enseñanza de la Ciencias Sociales sigue orientada por medio de la enseñanza de la Historia y la geografía, donde la primera está enfocada en cronología y periodos históricos representativos, y la segunda ofrece los marcos de referencia de localización de países, hecho que muestra que la enseñanza sigue siendo segmentada. Por eso, la enseñanza de las Ciencias Sociales integradas poco se evidencia en las aulas de clase, a pesar de que las reformas y contrarreformas lo hayan dispuesto así. De igual modo, se hace evidente que, a pesar de la integración, la formación docente, los textos escolares y las prácticas pedagógicas están dirigidas por el asignaturismo, lo que permite pensar que no se ha comprendido completamente el concepto de integración, pues las prácticas pedagógicas siguen siendo fragmentadas por disciplinas. 
En su proceso investigativo Fernández y Ochoa (2014), tuvieron como finalidad analizar la configuración histórica del diseño curricular de las Ciencias Sociales escolares en Colombia entre los años 1970 y 2010. Los investigadores concluyen que los Marcos Generales de los Programas Curriculares en Ciencias Sociales han evolucionado en comparación con los fijados en 1963, los cuales establecían la enseñanza de las Ciencias Sociales con base en la Historia y la Geografía de manera tradicional, debido a que las demás reformas curriculares realizadas le han apostado a una enseñanza de las Ciencias Sociales integradas, aunque aún en la práctica dista de una enseñanza desde el conocimiento holístico.

Se debe agregar también que Velasco (2014), pretendió hacer una aproximación a la producción sobre la Historia reciente de la enseñanza de las Ciencias Sociales en Colombia, para determinar los ámbitos en que se han investigado, así como los avances, limitaciones y vacíos que persisten en este. A partir de su investigación, confirma que quedan por completar espacios en los procesos de configuración y reconfiguración de las Ciencias Sociales, como lo son: la manera en que se concreta la enseñanza en los planes de estudio, libros de texto, programas, programaciones y acercamientos a las prácticas de enseñanza a través de memorias, informes, diarios, cuadernos de clase, etc., lo cual posibilitaría la creación de una línea de investigación dedicada a la Historia de las Ciencias Sociales escolares.

Concepciones de la enseñanza de las Ciencias Sociales en educación primaria en Colombia desde los referentes de calidad.

En el marco de la configuración de las Ciencias Sociales en Colombia fue la escuela primaria el campo de apropiación a principios del siglo XX donde emergió la enseñanza de la Historia, Geografía, la Antrogeografía y los Estudios Sociales como asignaturas y disciplinas con la finalidad de responder a la pregunta ¿Quiénes somos? y para establecer relaciones entre el pueblo colombiano, su cultura y el medio geográfico.

Dinámica que en los años sesenta y ochenta cambió debido a que el sistema educativo colombiano sufrió una revolución curricular, que para el caso de las Ciencias Sociales según Sánchez (2012), tomó forma a través del decreto 1002 de 1984, el cual convirtió a las Ciencias Sociales en un área de enseñanza obligatoria, para superar la enseñanza de la Geografía y la Historia de forma segmentada.

A su vez, en la década de los noventa, se da la promulgación de la Ley general de Educación de 1994, la cual integra las Ciencias Sociales, la Historia, la Geografía, la Constitución Política y la Democracia como áreas obligatorias, donde en su artículo 23 le atribuye el objetivo de buscar el conocimiento y comprensión de la realidad nacional para consolidar los valores propios de la nacionalidad colombiana tales como la solidaridad, la tolerancia, la democracia, la justicia, la convivencia social, la cooperación y la ayuda mutua. Por eso, para la básica primara en su artículo 21 plantea que se debe formar a los niños en asuntos como: fomento de los valores fundamentales para la convivencia en una sociedad democrática, participativa y pluralista; en la comprensión básica del medio físico, social y cultural en el nivel local, nacional y universal; en la formación para la participación y organización infantil; en el desarrollo de valores civiles, éticos y morales; en la iniciación y conocimiento de la Constitución Política y la adquisición de habilidades para desempeñarse con autonomía en la sociedad.

Igualmente, en 1994 se expide el decreto 1860 que señala que las áreas pueden configurarse desde proyectos pedagógicos transversales, entre ellos el estudio de la comprensión y la práctica de la constitución y la educación para la justicia, la paz, la democracia, la solidaridad, la confraternidad, el cooperativismo y en general, la formación en los valores humanos, al tiempo que, en 1996 con la resolución 2343 se crean 
los indicadores de logro que se fijaron con fines evaluativos.

Más tarde, para darle cumplimiento a los requerimientos que había señalado la Ley general de educación de 1994, en el año de 1998, se inició con la construcción de los Lineamientos Curriculares de Ciencias Sociales cuándo el MEN convocó a un amplio número de académicos en el país con el fin de iniciar un debate con relación a cuáles deberían ser las orientaciones curriculares de esta área (Sánchez, 2012). Dichos lineamientos fueron conocidos en el año 2002, y con ellos se buscaba una enseñanza holística, integradora, flexible, abierta, interdisciplinar y en espiral, la cual se podría lograr mediante el uso de los problemas relevantes, ejes generadores y ámbitos conceptuales. Es por ello que dicho documento fue presentado como una manera de superar la enseñanza fragmentada y por disciplinas.

Ahora bien, para poder llevar a cabo lo planteado con los Lineamientos Curriculares, en el año 2004 se publican los Estándares Básicos de Competencia de Ciencias Sociales, (EBC), que se encuentran organizados en los siguientes ejes: me aproximo al conocimiento como científico social; manejo conocimiento propios de las Ciencias Sociales y desarrollo de compromisos personales y sociales. Es necesario agregar que del eje conocimiento propios de las Ciencias Sociales, se desprende las relaciones con la Historia y la cultura, relaciones espaciales y ambientales y relaciones ético-políticas. Asimismo, se debe mencionar que estos agrupan las competencias por grupos de grados.

Como una manera de responder a los Lineamientos Curriculares y Estándares Básicos del área, el MEN en el año 2016 expide el documento Derechos Básicos de Aprendizaje (DBA) los cuales se organizan a través de cinco ejes articuladores que son: espacialidad, temporalidad, culturalidad, institucionalidad y DDHH desde las lógicas transdisciplinarias que reúnen los estudios culturales, los enfoques decoloniales y poscoloniales, los estudios de género y el feminismo, los estudios socio espaciales y los estudios críticos del desarrollo.

Se puede decir entonces que los Lineamientos Curriculares de las Ciencias Sociales, al ser el fundamento teórico, conceptual y epistemológico, se convierten en un referente necesario de retomar por los DBA para fortalecer así la mirada interdisciplinar en el proceso de enseñanza y aprendizaje. Por su parte, los EBC son el documento donde se plasma las competencias necesarias para interpretar el mundo, tanto para la básica como para la media, por ello los DBA están en coherencia con los Lineamientos Curriculares y los Estándares para que haya una lógica entre lo teórico, las competencias y el aprendizaje. Así mismo los DBA retoman de los EBC el conocimiento como científico social.

La guía de aprendizaje como dispositivo donde se materializa la enseñanza de las Ciencias Sociales.

Acerca de la guía de aprendizaje como dispositivo donde se materializa la enseñanza es necesario identificar qué procesos investigativos se han realizado sobre este asunto y a qué conclusiones se han llegado.

En relación con este punto, se encuentra que García y de la Cruz (2014), realizaron un estudio con el fin de compendiar información sobre la importancia de la guía didáctica en los procesos de aprendizaje, ya que es un medio para el desarrollo de la autonomía, habilidades, destrezas y aptitudes, y además le permite al estudiante hacer una autoevaluación de su proceso, convirtiéndose así en un elemento mediador entre estudiante y profesor.

Por esta misma línea continua Marcos (2016), quien en su estudio buscó analizar el caso de la enseñanza del patrimonio cultural a través de las guías didácticas. Allí encontró que este material es de gran ayuda para los docentes a la hora de desarrollar procesos propios de las asignaturas, 
puesto que posibilita el trabajo autónomo por parte de los estudiantes.

En consonancia con lo anterior, Pino y Urías (2020), realizaron una reflexión sobre los aspectos teóricos y prácticos relacionados con las guías didácticas y pudieron concluir que estas son un recurso significativo, pues permiten un óptimo desarrollo de la enseñanza y el aprendizaje, más aún en la época de pandemia por COVID-19, pues son una herramienta que se puede adaptar a diferentes contextos y grupos étnicos, a diversas propuestas curriculares, trabajo por competencias, por asignaturas o por disciplinas. Para una correcta elaboración de guías, es importante tener en cuenta algunos elementos estructurantes como lo son: título, introducción, descripción del contenido, objetivos, actividades, evaluación (autoevaluación, heteroevaluación, coevaluación) y bibliografía.

En definitiva, se encuentra que las investigaciones que se han realizado sobre la enseñanza de las Ciencias Sociales en los últimos cincos años, han tendido diferentes perspectivas. En primer lugar, se encuentran las didácticas donde se resalta el uso de los textos escolares o libros de textos que se apoyan en la historia para reproducir la idea de Estado-Nación y el ideal ciudadano a formar, por eso la necesidad de que este material promueva contenidos sociales por medio de diferentes procedimientos para así fomentar la interpretación y la reflexión. También evidencia que la reflexión crítica en la escuela primaria no se ha dado debido a que el docente no cuenta con la preparación adecuada para abordar este asunto, lo que genera que en ocasiones se centren las actividades en el alumnado. De ahí que se haga necesario que los docentes aborden problemas relevantes en el proceso de enseñanza, puesto que es una manera de analizar y descubrir posibles soluciones para generar un cambio social; igualmente, se deben generar prácticas de enseñanza basadas en la interacción social con el fin de construir procesos significativos.

Algunas investigaciones se enfocan en distintas disciplinas sociales con un corte didáctico, entre ellas sobresalen disciplinas como Historia y Geografía con prácticas tradicionales alejadas de la realidad, centradas en libros de textos que le dan un rol pasivo al estudiante y que no le permite profundizar los conceptos propios de cada disciplina, lo que pone en tela de juicio la rigurosidad epistemológica. Lo anterior da a entender que no se aprovechan lo suficiente los conceptos propios y los procedimientos, los cuales tienen una riqueza didáctica para que se pueda comprender la realidad social desde diferentes perspectivas.

En segundo lugar, se identifican estudios históricos que se han preocupado por la configuración de las Ciencias Sociales, a partir de ellos se ha logrado vislumbrar que esta ha pasado por enfoques civilizatorios, desarrollistas, cientificistas, mercantilistas, por competencias, y por prácticas tradicionales y reproductoras. También demuestran que las reformas curriculares que han ocurrido en Colombia han puesto los contenidos como una forma de ejercer control y que, a pesar de las reformas y contrarreformas, aún no se puede hablar de una integración de las Ciencias Sociales, pues prima el trabajo segmentado y por disciplinas. Por eso es de vital importancia que, para indagar por la configuración de la enseñanza de las Ciencias Sociales, los investigadores centren su mirada en la materialización, es decir, en los planes, los programas, los informes, los cuadernos, las guías y los módulos.

\section{Posibles alternativas de solución.}

Después de haber hecho un recorrido por lo que se ha venido investigando con respecto a la enseñanza de las Ciencias Sociales, es necesario buscar que las prácticas de enseñanza en la escuela primaria trasciendan del enfoque tradicional y reproductivo, por lo cual se hace necesario que los docentes que orientan los procesos de esta área en primaria cuenten con una formación adecuada para poder profundizar los conceptos propios de cada disciplina y su aplicabilidad en el contexto. 
Es de vital importancia también, promover una enseñanza de las Ciencias Sociales de forma integrada y no por disciplinas segmentadas, ya que así, se puede lograr formar estudiantes críticos y reflexivos, que generen cambios y transformaciones en el mundo, es decir, convertirlo en un mundo más justo, equitativo y solidario, lo cual se puede lograr mediante el trabajo por ejes generadores tal como lo plantea los Lineamientos Curriculares, para ello, también se pueden emplear preguntas problematizadoras que partan de los intereses y necesidades de los estudiantes y que permitan el uso de diferentes competencias y contenidos para entenderla desde una perspectiva multicausal. Dicho de otro modo, al hacer uso de configuraciones didácticas como las investigaciones y proyectos de aula, se puede obtener que el estudiante tenga un rol activo, vaya más allá de lo establecido, asuma una posición analítica y reflexiva frente a los hechos, haga uso de conceptos propios de las Ciencias Sociales para comprender lo que ocurre y con ello promueva un mundo más justo y equitativo.

Por eso se hace necesario que las investigaciones busquen comprender la enseñanza de las
Ciencias Sociales en la primaria, pues es un espacio singular donde la asignación de las áreas no se hace mediante la especificidades de los docentes, sino de acuerdo con las necesidades de la institución, de ahí que los docentes, para poder cumplir con la enseñanza de esta área, hacen uso de diferentes elementos como seguir de forma rigurosa lo que establece el plan de área, los referentes de calidad o libros de texto.

Por otro lado, es valioso identificar cómo la configuración se concretiza a través de los cuadernos, libros de textos, planes de áreas, informes, planeadores, guías de aprendizajes y módulos, es decir, salirse de investigaciones didácticas, históricas o las centradas en macrocurrículos y microcurrículos e ir a la escuela para comprender qué sucede en ella, cómo se trabajan los diferentes contenidos y con qué finalidad, ya que esto posibilitará que el maestro realice reflexiones sobre su quehacer pedagógico, además, es muy posible que se motive a transformar sus procesos de enseñanza y aprendizaje y se reconozca que existen diferentes configuraciones didácticas que pueden ser de mayor interés para el estudiante.

\section{REFERENCIAS}

1. Aguilera, A. (2016). La enseñanza de la Historia y las Ciencias Sociales hoy: contrasentidos y posibilidades. Folios, Segunda época, 46 (2017). 15-27. https://doi.org/10.17227/01234870.46fo lios 15.27

2. Amézola, G y D’Archary, C. (2016). La Dictadura Congelada. Actos escolares, clases conmemorativas y carteleras sobre la última dictadura militar argentina en escuelas primarias de la Provincia de Buenos Aires (2008-2015). Revista Colombiana de Educación, (71), 137-161. https:// doi.org/10.17227/01203916.71rce137.161

3. Díaz, N. (2017). Problemas sociales relevantes en el aula de primaria: la "cartografía de la controversia" como método, Revista de Investigación en Didáctica de las Ciencias Sociales 1 (2017), p. p. 24-38. https://doi.org/10.17398/2531-0968.01.24

4. Duque, L. (2017). Conflicto social colombiano: representación en textos escolares de Ciencias Sociales. magis, Revista Internacional de Investigación en Educación, 9 (19), 49-68. doi: 10.11144/ Javeriana.m9-19.csc

5. Fernández, O y Ochoa, J. (2014). Planteamientos discursivos en torno a las reformas que incidieron en el diseño curricular de las Ciencias Sociales escolares en Colombia (1970- 2010). Revista Virajes, 16 (2). 275-296. http://vip.ucaldas.edu.co/virajes/downloads/Virajes16(2)_13.pdf

6. García, H y De la Cruz. (2014). Las guías didácticas: recursos necesarios para el aprendizaje autónomo. Edumecentro, 6 (3), 165-175. http://scielo.sld.cu/scielo.php?script=sci_arttext\&pid $=\mathrm{S} 2077-28742014000300012$

7. Gutiérrez, M y Arana, D. (2014). "La formación del pensamiento social en la enseñanza y el 
aprendizaje en la educación básica primaria". Revista Latinoamericana de Estudios Educativos, No. 2, Vol. 10. 124-144. Manizales: Universidad de Caldas. https://www.semanticscholar.org/paper/ LA-FORMACI\%C3\%93N-DEL-PENSAMIENTO-SOCIAL-EN-LA-ENSE\%C3\%91ANZAGuti\%C3\%A9rrez-Arana/a7fcac1398048f22ebe01d56e4a5b9aef89718db

8. Gómez, A, Sevilla, J y Fernández, R. (2015). Hacer emerger la diversidad de espacios y los lugares vulnerables a través de un recurso de escasa visibilidad en el currículo de educación primaria: la toponimia. En una enseñanza de las Ciencias Sociales para el futuro: recursos para trabajar la invisibilidad de personas, lugares y temáticas. AUPDCS. 183-192. https://dialnet.unirioja.es/ servlet/libro? codigo $=570009$

9. Hernández, L. (2014). La enseñanza y el aprendizaje del tiempo histórico en la educación infantil. Las representaciones sociales y la práctica docente de una estudiante de maestra de educación infantil. Enseñanza de las Ciencias Sociales, 2014 (19). 31-39. https://www.raco.cat/index.php/ EnsenanzaCS/article/view/285717

10. Marcos, M. (2016). La guía didáctica: herramienta de enseñanza del patrimonio y los bienes culturales. 32 (11), 856-872. https://www.redalyc.org/articulo.oa?id=31048902050

11. Martínez, A. (2003). Currículo y modernización cuatro décadas de educación en Colombia. http:// www.albertomartinezboom.com/escritos/libros/2003_Curriculo_y_Modernizacion.pdf

12. MEN. (1994). Decreto 1860 de agosto de 1994. por el cual se reglamenta parcialmente la Ley 115 de 1994, en los aspectos pedagógicos y organizativos generales. https://www.mineducacion. gov.co/1759/w3-article-86240.html?_noredirect=1\#: :text=Por\%20el\%20cual\%20se $\% 20$ reglamenta,aspectos $\% 20$ pedag $\% \mathrm{C} 3 \% \mathrm{~B} 3$ gicos $\% 20 \mathrm{y} \% 20$ organizativos $\% 20$ generales.

13. MEN (1996). Resolución 2343 de junio 5 de 1996. Por la cual se adopta un diseño de lineamientos generales de los procesos curriculares del servicio público educativo y se establecen los indicadores de logros curriculares para la educación formal. https://repository. usta.edu.co/bitstream/handle/11634/280/RESOLUCION_2343_DE_JUNIO_5_DE_1996. pdf? sequence $=21 \&$ is Allowed $=y$

14. MEN (2002). Lineamientos curriculares de Ciencias Sociales, https://www.mineducacion.gov. co/1759/articles-339975_recurso_1.pdf

15. MEN (2004). Estándares básicos de competencia de Ciencias Socialeshttps://www.mineducacion. gov.co/1621/articles-116042_archivo_pdf3.pdf

16. MEN (2016). fundamentos de Derechos Básicos de Ciencias Socialeshttps://aprende. colombiaaprende.edu.co/ckfinder/userfiles/files/fundamentacioncienciassociales.pdf

17. MEN (2016). Derechos Básicos de Aprendizaje- Ciencias Socialeshttp://aprende.colombiaaprende. edu.co/sites/default/files/naspublic/DBA_C.Sociales.pdf

18. Moreno, O. (2018). La enseñanza de las Ciencias Sociales: un diagnóstico a partir de las memorias, reflexiones y expectativas de profesores de enseñanza primaria en formación inicial, Ensaio: aval. pol. públ. Educ., 26 (100). 1021-1037. https://doi.org/10.1590/S0104-40362018002601452

19. Ortega, D. (2015). Didáctica de la Historia y construcción de la identidad cultural iberoamericana en el currículo y libros de texto de Ciencias Sociales de Educación Primaria españoles. Repositorio institucional universidad de Burgos, http://hdl.handle.net/10259/3746

20. Pino, R y Urías, G. (2020). Guías didácticas en el proceso enseñanza-aprendizaje: ¿Nueva estrategia? Revista Scientific, 5(18), 371-392. https://doi.org/10.29394/Scientific.issn.25422987.2020.5.18.20.371-392

21. Pinochet, S y Pagès, J. (2016). ¿Cuál es el protagonismo de los niños, las niñas y los jóvenes en el currículo de Historia, Geografía y Ciencias Sociales de Chile? El impacto del currículo en las ideas de los niños y de los jóvenes sobre su protagonismo en la Historia, Práxis Educativa 11 (2), pp. 1-20. http://www.redalyc.org/articulo.oa?id=89442687004

22. Pinto, E y Molina, S. (2015). La educación patrimonial en los currículos de Ciencias Sociales en España y Portugal Educatio Siglo XXI, 33 (1). 103-128. https://doi.org/10.6018/j/222521

23. Presidencia De La Republica (1994, 3 de agosto). Decreto 1860 de 1994, Por el cual se reglamenta parcialmente la Ley 115 de 1994, en los aspectos pedagógicos y organizativos generales. Diario Oficial No 41.473. https://www.mineducacion.gov.co/1621/articles-172061_archivo_pdf_ decreto1860_94.pdf

24. Romero, M y Crisol. (2021). Las guías de aprendizaje autónomo como herramienta didáctica de apoyo a la docencia. Escuela abierta 15(1), 9-31. https://dialnet.unirioja.es/servlet/ articulo? codigo $=4078711$ 
25. Sáez-Rosenkranz I. (2017). La enseñanza de la Historia en los libros de texto de educación básica en Chile. History teaching in primary school textbooks in Chile, enseñanza de las Ciencias Sociales, 2017 (16). 27-40. Http://www.publicacions.ub.edu/revistes/eccss 16/default.asp?Articulo=1390

26. Sánchez, N. (2012). Las Ciencias Sociales escolares entre 1984-2010 en Colombia [tesis de maestría, Universidad de Antioquia] repositorio UdeA, http://ayura.udea.edu.co:8080/jspui/ handle/123456789/1500.

27. Silva, O. (2018). Investigar históricamente las disciplinas y los saberes escolares: el caso de la enseñanza de las Ciencias Sociales en Colombia. Pedagogía y Saberes, 49, 81-9.

28. Tosar, B. (2018). Literacidad crítica y enseñanza de las Ciencias Sociales en primaria: "Profe, las bolsas de plástico no son medusas" REIDICS 2 (2018), 4-19. https://doi.org/10.17398/25310968.02.4

29. Velasco, G. (2014). La Historia de la enseñanza de las Ciencias Sociales como referente para la transformación crítica de las prácticas educativas, Uni-pluri/versidad, 14 (1). 78-89. https:// revistas.udea.edu.co/index.php/unip/article/view/19819

30. Velasco, G. (2017). Las reformas al currículo oficial: la configuración de las Ciencias Sociales escolares en la educación secundaria en Colombia (1939-1974), Uni-pluri/versidad, 18, (1). 78-89. 1017533/udea.unipluri.18.1.08

31. Zuluaga, O. (1989). Pedagogía e Historia: la historicidad de la pedagogía, la enseñanza, Un objeto de saber, Santa fe de Bogotá: Siglo del Hombre Editores, Anthropos, Editorial Universidad de Antioquia p. p 212 